\title{
Spherical wrist dimensional synthesis adapted for tool-guidance medical robots
}

\author{
T. Essomba ${ }^{1,2}$, L. Nouaille ${ }^{1}$, Med Amine Laribi $^{2}$,a, C.A. Nelson ${ }^{3}$, S. Zeghloul $^{2}$ And \\ G. POISSON ${ }^{1}$ \\ 1 PRISME Laboratory, Université d'Orléans, INSA CVL, France \\ 2 PPRIME Institute, Dept. GSMC - CNRS ENSMA UPR 3346, University of Poitiers, France \\ 3 Dept. of Mechanical and Materials Engineering, University of Nebraska-Lincoln, USA
}

Received 20 June 2013, Accepted 19 March 2014

\begin{abstract}
The objective of this article is to present the dimensional synthesis of serial and parallel spherical wrists, an important step in the design process of medical robots. This step is carried out to obtain optimal dimensions of tool-guidance medical robots. With this goal, we have first studied the specifications of two robots with different medical applications: one for tele-echography examination and one for minimally invasive surgery. Then, we have established that the medical needs expressed by the doctors were very different but the specifications in robotic terms have a lot of common points (kinematics, workspace, bulkiness). For both applications studied, robots need a mobility of three rotations around a fixed point (probe contact point on the patient's skin or trocar incision). So, a spherical wrist architecture is adapted to their needs. An important constraint related to medical applications is that the robot must be compact in order to not obstruct or collide with its environment (medical personnel or patient). We perform dimensional synthesis allowing determination of dimensions of the mechanism for serial and parallel spherical wrists, for a tele-echography robot, and a serial wrist for a minimally invasive surgery robot. We use multicriteria optimization methods minimizing a cost function to obtain both good kinematic performance and compactness for the architecture. The difficulty/challenge of this design process, depending of the studied applications, is the choice of efficient criteria describing the performances and the constraints of the robot. The design variables must faithfully represent the specifications of the robot so that its performance can respond to the medical requirements. We show, here, the different methods used for optimizing the chosen kinematic architecture for the particular medical application. These studies lead to prototypes which are validated after medical experiments. This process of dimensional synthesis will be applied to other medical applications with different sets of specified constraints.
\end{abstract}

Key words: Tool-guidance medical robot / dimensional synthesis / spherical parallel manipulator / spherical serial robot / optimization

\section{Introduction}

Medical robotics is a recent discipline. In the two last decades, a number of medical robot prototypes have been created, but they are designed to respond to precise tasks in various medical applications. Their kinematic architectures have evolved and their capabilities have improved. The problem is that the medical robots often remain as prototypes, while few robots are used in real medical applications. The most successfully commercialised is the $d a$ Vinci robot used in minimally invasive surgery.

\footnotetext{
a Corresponding author:

med.amine.laribi@univ-poitiers.fr
}

This observation leads us to be interested in studying the dimensional synthesis step for medical robots used in different medical applications. First, we detail the medical applications studied - tele-echography and minimallyinvasive surgery - and present the specifications of the two medical robots. We point out the common specifications and the different points between the two medical applications. Then, we present the methods used for the dimensional synthesis. Finally, we compare the different approaches to develop solutions to carry out dimensional synthesis for other medical robots.

Several studies on optimizations of spherical mechanisms have already been carried out. A serial spherical wrist mechanism, used for minimally invasive surgery, was 
Table 1. Tele-echography robot and minimally-invasive robot specifications.

\begin{tabular}{|c|c|c|}
\hline & Tele-echography robot & MIS robot \\
\hline Kinematic & $\begin{array}{l}4 \text { DoF ( } 3 \mathrm{R} \text { with } \mathrm{RCM}+T) \\
\text { conical workspace } \theta=35^{\circ} \\
\text { avoid singularities }\end{array}$ & $\begin{array}{l}4 \text { DoF (3R with RCM }+T) \\
\text { conical workspace } \theta=60^{\circ} \\
\text { avoid singularities }\end{array}$ \\
\hline Forces and Torques & $F=20 \mathrm{~N}$ & $F=20 \mathrm{~N}$ \\
\hline Safety constraints & No collision between the moving parts of the robot & $\begin{array}{l}\text { No collision between the moving parts } \\
\text { of the robot }\end{array}$ \\
\hline & No collision with the patient's body $\left(\theta_{\mathrm{s}}=75^{\circ}\right)$ & No collision with the patient's body \\
\hline Specific constraints & Transportable to isolated sites, body mounted & $\begin{array}{l}\text { Transportable to different operating } \\
\text { rooms }\end{array}$ \\
\hline Dimensions & Lightweight: mass (less than $3 \mathrm{~kg}$ ), compact: width $<45 \mathrm{~cm}$ & Compact and lightweight \\
\hline
\end{tabular}

optimized by Lum using a cost function depending on the mechanism isotropy and stiffness $[1,2]$. Gosselin optimized a 3 DoF spherical parallel manipulator to obtain an isotropic robot [3]. Other authors sought optimum design of parallel spherical manipulators considering only dexterity $[4,5]$. Chaker et al. investigated design and analysis of a spherical parallel mechanism (SPM) to be used as a haptic device for a medical application. An optimization procedure was carried out to find the optimal spherical manipulator with the closest workspace to the desired one $[6,7]$.

The particularity of our optimization process is taking into account two opposite criteria (kinematic performance, size) to make a design compromise to obtain a compact, lightweight robot, having good path following needed in medical robotics.

\section{Medical robot specifications}

The medical needs of tele-echography robots and minimally-invasive robots are detailed. Then, the specifications of the two medicals robots are described.

\subsection{Echography robot}

Echography is an imaging technique widely used in all kinds of examinations (abdominal, cardiac, foetal or emergency diagnostics). This technique presents the advantages of being rapidly implemented and inexpensive. The result of the diagnosis can be immediately given to the patient. But this technique is expert dependent and the societal problem is that there is a lack of experts in regional hospitals or in isolated sites. A tele-echography robot then allows a medical expert to perform this clinical act on a patient at a distance. In the tele-operated practice, the doctor operates a fictive probe in the "expert premises", as shown in Figure 1. The probe movements are sent to a slave robot situated at the "patient premises" by satellite or terrestrial links. The robot is designed to hold and move the real ultrasound probe on the patient's body and reproduce the fictive probe movement to the real one. Ultrasound images are sent back from the patient site to the medical expert who can perform his diagnosis, in real time, just as he would perform a real echography examination.

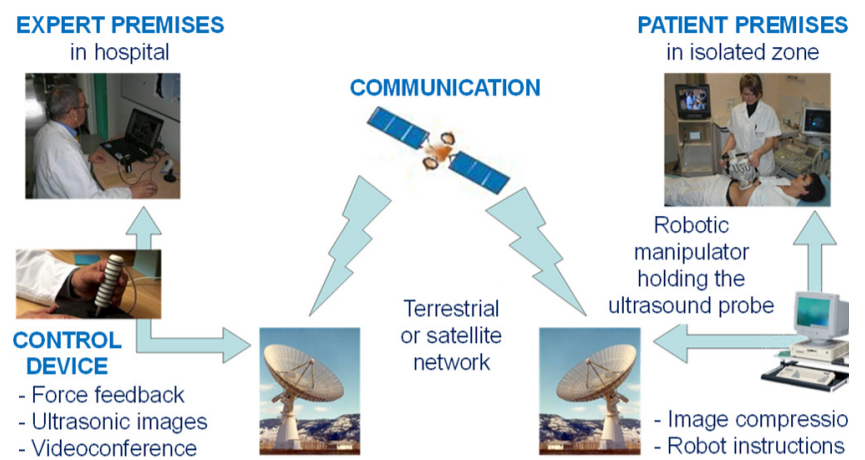

Fig. 1. Tele-echography robot principle.

\subsection{Minimally-invasive surgery (MIS) robot}

The practice of surgery has progressed in the last two decades from an invasive paradigm to a primarily minimally invasive paradigm. Surgeons now use several long, thin tools, including a camera, to perform many different types of abdominal surgery. Robotics can be applied to MIS to enable expert telesurgery, remove tremor, provide motion scaling, and so forth. Therefore, the objective is to create a compact telesurgical robot which conforms to the constraints imposed by the MIS paradigm.

\subsection{Medical intervention requirements}

After having studied the medical gestures with different approaches and different medical experts [8-10], the specifications of the two medical robots are established. They are summarized in Table 1.

The specifications describing kinematics, forces and torques, safety constraints, dimensions and specific constraints are detailed. Many common points between the two medical specifications can be mentioned as follows. The kinematic specifications are similar. The two robots need 4 DoF, 3 rotations with a remote centre of motion $(\mathrm{RCM})$ and one translation to exert a force along the tool axis. The RCM is the contact point between the probe and the patient skin for the tele-echography robot and the trocar incision for the MIS robot. The workspace is defined by a cone. The cone angle of the cone depends on the application (about $35^{\circ}$ for tele-echography and $60^{\circ}$ for MIS). 
These values, determined by the medical gestures analysis, have fuzzy limits, because the gesture highly depends on the expert use. Singularities inside the workspace must be avoided to obtain good path following. The maximum force exerted by the effector is fixed at $20 \mathrm{~N}$ for the two robots, one to push the probe to obtain a better echography image of the organ, the other for using the MIS tools. The safety constraints are very important in medical robotics. The robot must have no collision with the patient's body. A safety angle has been defined to respect this condition $\left(\theta_{\mathrm{S}}=75^{\circ}\right.$ for tele-echography).

The robots present specific needs which depend on the application. The tele-echography robot must be transportable to different sites/premises, and supported by the patient. These needs lead to dimensional constraints. The tele-echography robot must have a mass less than $3 \mathrm{~kg}$. It must be compact; its width must be less than $45 \mathrm{~cm}$ so as to not be in the way of the patient. The MIS robot must be transportable to different operating rooms, so it must be compact and lightweight as well. So, the specifications are very similar, with only the limit of the conical workspace being significantly different. The first specification we must respect is the robot mobility. The kinematic architecture chosen allows satisfaction of this constraint.

\subsection{Topology specifications}

The robots must both have 3 rotations with a RCM. The naturally suited structural candidate is the serial spherical wrist. However, we also consider the parallel spherical wrist for the tele-echography robot because this architecture, according the kinematic choices, may not have singularity in the workspace. The kinematic architectures which are to be dimensionally optimized are presented in Figure 3.

The vector of the design variables, $\mathrm{I}_{a, b, c}$, for each architecture is defined as follows:

- $\mathrm{I}_{a}=\left[\beta_{3} \beta_{5} r L\right]^{\mathrm{T}}$ for the serial minimally invasive surgery robot where $\beta$ are link angles of the serial wrist, $r$ is the effective radius of the outermost link in the wrist, and $\mathrm{L}$ is the distance of that outermost link from the remote centre of rotation.

- $\mathrm{I}_{b}=\left[\alpha_{0} \alpha_{1} \alpha_{2}\right]^{\mathrm{T}}$ for the serial tele-echography robot where $\alpha_{i}$ are angles between $z_{i}$ and $z_{i+1}$ axes (see Fig. 3b).

- $\mathrm{I}_{\mathrm{c}}=[\alpha \beta \gamma \xi]^{\mathrm{T}}$ for the parallel tele-echography robot where $\alpha$ characterizes the first link and the angle $\beta$ the second link of each serial $\underline{R R R ~ l e g . ~ T h e ~ a n g l e ~} \gamma$ defines the angle between the axis $Z_{\mathrm{E}}$ normal to the moving platform and the last joint axis. The base is composed of a rigid support which orients the first revolute of each leg with angle $\xi$.

\section{Dimensional synthesis}

The aim of this section is to develop and to solve a multidimensional, nonlinear optimization of selecting the geometric design variables for the tool-guidance robot. The robot must be compact and describe a desired workspace with good kinematic performance.

\subsection{Optimization formulation}

The optimal dimensional synthesis of the toolguidance robot can be defined as follows:

- Given a specific workspace to be reached.

- Find the optimal parameters of the tool-guidance robot to reach the specific workspace with suitable kinematic performances while remaining compact.

For each robot architecture a specific optimization problem is stated in order to obtain the optimal design vector I. This vector minimizes or maximizes an objective function, $\mathrm{F}(\mathrm{I})$. The functions and/or the constraints, used for each architecture, include workspace reaching, the enhancement of the dexterity and the compactness.

These specifications are listed in the previous section.

In the next section, we detail the formulation of the optimization for serial and parallel wrists. The formulation differs from one architecture to another - and from one application to another - but the approach remains the same. The difference between the methods used for each robot optimization rests on the criteria and the constraint choices. The challenge is to formulate the optimisation which properly reflects the medical experts' needs.

\subsection{Serial spherical wrist}

\subsubsection{MIS application}

The robot must describe a conical workspace with good kinematic performance and must be compact. A positive objective function $\mathrm{F}\left(\mathrm{I}_{a}\right)$ is proposed to linearly combine all criteria and is used to evaluate candidate solutions. The overall objective function to evaluate the performance is:

$$
\mathrm{F}\left(\mathrm{I}_{a}\right)=k_{1} W+k_{2} K_{g}+k_{3} S
$$

where $k_{i}$ is the weight of the $i$ th criterion and $k_{i}$ such that $k_{1}+k_{2}+k_{3}=1$. The considered criteria, including workspace $(W)$, consistent manipulability $\left(K_{g}\right)$ and size $(S)$, are minimized to achieve desirable performance.

The consistent manipulability is defined by the following expression [8]:

$$
K_{g}=1-\left(1-D_{g}\right)\left(1-U_{\sigma}\right) \quad K_{g} \in[0,1]
$$

where $D_{g}$ (average sampled $D_{l}$ ) accounts for performance across the workspace and $U_{\sigma}$ (uniformity) accounts for the uniformity of that performance:

$$
U_{\sigma}=\sqrt{\frac{1}{N} \sum_{i=1}^{N}\left(\frac{D_{l_{i}}-D_{g}}{D_{l_{\max }}-D_{l_{\min }}}\right)^{2}} \quad U_{\sigma} \in[0,1]
$$




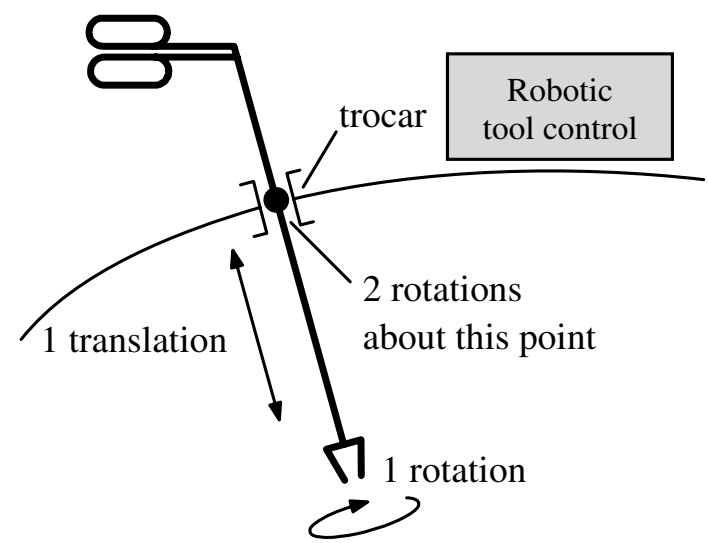

Fig. 2. Minimally invasive robot principle.

and

$$
D_{l}=\frac{1}{1+\sqrt{\operatorname{det}\left(J J^{T}\right)}}, \quad D_{l} \in[0,1]
$$

The workspace index is defined as follows [8]:

$$
\mathrm{W}=\left\{\begin{array}{ll}
\frac{\phi_{\mathrm{c}}-\Delta \phi}{\phi_{\mathrm{c}}} & \text { if } \phi_{\text {out }} \leqslant \phi_{\mathrm{c}} \\
\frac{\phi_{\mathrm{in}}}{\phi_{\mathrm{c}}} & \text { if } \quad \phi_{\text {out }}>\phi_{\mathrm{c}},
\end{array} W[0,1]\right.
$$

where $\phi_{\mathrm{c}}$ is the constraint on the inclination angle of the manipulator.

The size index is defined as follows [8]:

$$
S=\frac{R_{\mathrm{p}}-R_{d}}{R_{\mathrm{p}}} ; \quad \mathrm{S} \in[0,1]
$$

where $R_{\mathrm{p}}$ is the physical radius of the spherical manipulator, and $R_{\mathrm{d}}$ is the constraint of minimal radius.

Each of these criteria is expressed such that the values can range from 0 to 1 , with 0 being optimal. By changing the priorities of each criterion, various desirable performances with different emphases can be achieved. The optimization goal is then to find a set of design parameters which minimizes the objective function according to the priorities expressed by the weights. A Genetic Algorithm (GA) method is used with the fitness function, $\mathrm{F}\left(\mathrm{I}_{a}\right)$, and allows avoidance of local minima. The obtained optimal robot design variables are presented in Section 4 . The final design was achieved using $k_{1}=0.2, k_{2}=0.6$, and $k_{3}=0.2$.

\subsubsection{Echography application}

The kinematic architecture adapted for teleechography is the serial spherical wrist. This architecture presents a singularity located at the workspace centre, when the first and the third axes are aligned. To displace the central singularity of the classic architecture outside the most frequently used part of the workspace, an incline offset of an angle $\alpha_{0}$ from the normal direction to the patient's skin is proposed in Figure 2.
The dimensional synthesis is carried out via an optimization procedure. The aim is to determine the design vector, I, of the inclined architecture to produce good kinematic performance near the singularity to offer better medical gesture following, as well as a compact architecture. The choice of the design criteria is fundamental for respecting the tele-echography robot specifications.

The optimal solution must have both good kinematic performance and compactness, respectively expressed using global dexterity $\eta$ and global compactness $C_{\mathrm{g}}$. The optimization for a suitably chosen objective function $\mathrm{F}\left(\mathrm{I}_{b}\right)$ is defined as:

$$
\begin{array}{cc}
\operatorname{maximize} & \mathrm{F}\left(\mathrm{I}_{b}\right)=k C_{\mathrm{g}}+(1-\mathrm{k}) \eta_{\mathrm{g}} \\
\text { Subject to } & \alpha_{1}+\alpha_{2}-\alpha_{0} \geqslant \theta_{n} \\
& \alpha_{1}+\theta_{n}<75^{\circ}
\end{array}
$$

where $\eta_{g}=\frac{\int_{W}\left(\frac{1}{K}\right) \mathrm{d} w}{\int_{W} \mathrm{~d} w}$ with $\frac{1}{K}=\frac{\sigma_{\min }}{\sigma_{\max }} \sigma$ is the set of eigenvalues of the matrix $J_{\omega} J_{\omega}^{\mathrm{T}}$ and $J_{\omega}$ is the rotation Jacobian matrix. $K$ is a condition number of the Jacobian matrix.

Also

$$
C_{\mathrm{g}}=\frac{\int_{W} C \mathrm{~d} w}{\int_{W} \mathrm{~d} w} \quad \text { with } \quad C=1-\max \left(\alpha_{0}, \delta\right) / \theta_{S}
$$

$\delta$ represents the incline of the $z_{2}$ axe (Fig. 3b), then $\max (\alpha, \delta)$ represents the maximal incline that the robot can have on the workspace, and $\theta_{\mathrm{s}}$ the safety angle defined by the safety specifications $\left(\theta_{\mathrm{s}}=75^{\circ}\right)$.

The maximum of the objective function is sought, with the weighting factor $k$ being a real number between 0 and 1 . The set of solutions is limited by the tele-echography robot constraints. The first constraint enforces the necessary workspace:

$$
\alpha_{1}+\alpha_{2}-\alpha_{0} \geqslant \theta_{n}
$$

The second constraint equation represents the safety angle condition:

$$
\alpha_{1}+\theta_{n}<\theta_{s}
$$

The choice to take into account the necessary workspace and the security angle as constraints and not as criteria is to guarantee these conditions are verified by the optimal robot. The conical workspace must have a minimal vertex angle $\theta_{n}=35^{\circ}$ and the robot must not be inclined more than $\theta_{\mathrm{s}}=75^{\circ}$ to safely ensure collision avoidance with the patient. The algorithm used to solve this problem is the gradient descent method under MATLAB software. Due to the monotony of the objective function, $\mathrm{F}\left(\mathrm{I}_{\mathrm{b}}\right)$, this algorithm is well adapted to optimization.

\subsection{Parallel spherical wrist}

The architecture to be designed has to respond to several specifications: it has to be lightweight and compact, and it has to be able to generate the minimal necessary workspace. Also, a good trajectory tracking is required. 


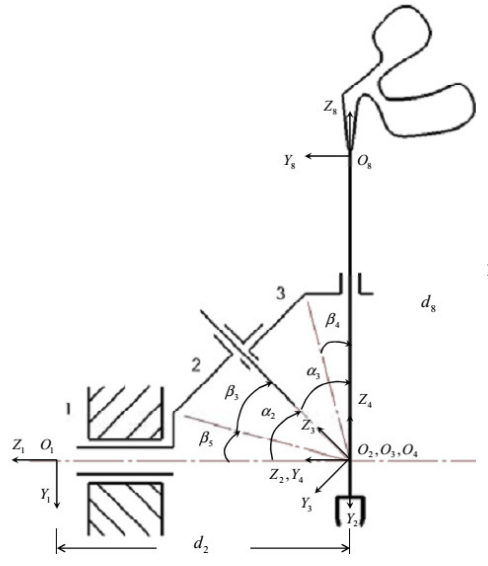

(a)

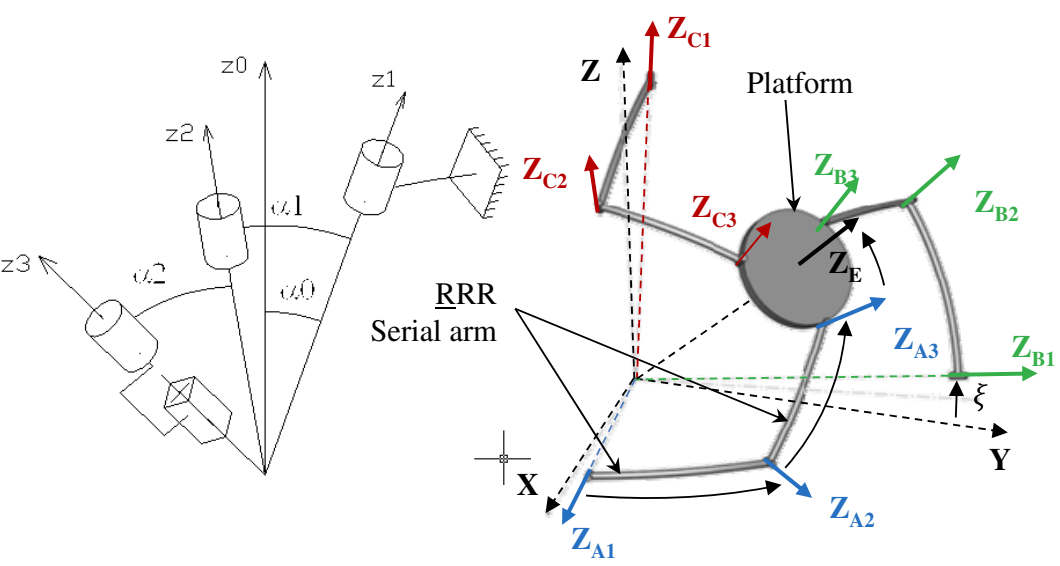

(b)

(c)

Fig. 3. Kinematical sketch and parameters of tool-guidance robots: (a) and (b) serial wrists; (c) parallel wrist.

The aim of the dimensional synthesis is to identify the design vector that will generate the highest dexterity level, considered as the criterion, while respecting the two selected constraints (workspace and compactness). A highest dexterity level allows motion within the workspace with equal ease in all directions. The associated optimization problem, with four parameters for a suitably chosen objective function $\mathrm{F}\left(\mathrm{I}_{\mathrm{c}}\right)$, can be stated as:

$$
\begin{array}{ll}
\operatorname{maximize} & \mathrm{F}\left(\mathrm{I}_{\mathrm{c}}\right)=\eta_{\mathrm{I}_{\mathrm{s}}}^{\mathrm{G}} \\
\text { Subject to } & \mathrm{W}_{\mathrm{I}_{\mathrm{c}}}=0 \\
& \mathrm{C}_{\mathrm{I}_{\mathrm{c}}}=0
\end{array}
$$

where the global dexterity is defined by : $\eta_{\mathrm{I}_{\mathrm{c}}}^{G}=\frac{\int_{\Omega} \frac{1}{\kappa(\mathrm{J})} \mathrm{d} w}{\int_{\Omega} \mathrm{d} w}$ and $\kappa(\mathrm{J})$ is the condition number of the SPM Jacobian matrix [11].

For a given architecture defined by the design vector $\mathrm{I}_{\mathrm{c}}$, the workspace constraint $W_{\mathrm{I}_{\mathrm{c}}}$ is defined by:

$$
\mathrm{W}_{\mathrm{I}_{\mathrm{c}}}=\sum_{\varphi=-\varphi_{W S}}^{\varphi_{W S}} \sum_{\psi=0}^{n} \sum_{\theta=0}^{m} P_{W}(\psi, \theta, \varphi)
$$

with $n=2 \pi / \Delta \psi, m=\theta_{w s} / \Delta \theta$, and $\theta_{W S}=35^{\circ}$ maximum angle. $\mathrm{P}_{W}(\psi, \theta, \varphi)$ is an incremental function with respect to $S_{k}, k \in\{A, B, C\}$. In the numerical tests $\Delta \psi$ and $\Delta \theta$ are set to $10^{\circ}$ and $1^{\circ}$ respectively.

$$
P_{W}(\psi, \theta, \varphi)=\left\{\begin{array}{c}
1 \text { if } S_{A, B, C}(\psi, \theta, \varphi)>0: \\
\text { outside the workspace } \\
0 \text { if } S_{A, B, C}(\psi, \theta, \varphi) \leqslant 0: \\
\text { within the workspace }
\end{array}\right.
$$

Here, the orientation power function $S_{k}$ indicates if the leg $\mathrm{k}$ is able to reach the given orientation or not [11]. $\mathrm{W}_{\mathrm{I}_{\mathrm{c}}}$ gives the number of unreachable points of the necessary workspace. A zero value of $\mathrm{W}_{\mathrm{I}_{\mathrm{c}}}$ means that the whole workspace is reachable.

$\mathrm{C}_{\mathrm{I}_{\mathrm{c}}}$ represents the number of orientations of the end effector that generates external collisions. When $\mathrm{C}_{\mathrm{I}_{\mathrm{c}}}$ is zero, the entire workspace is collision free. This constraint can be written as follows:

$$
C_{I_{\mathrm{c}}}=\sum_{\varphi=-\varphi_{w s}}^{\varphi_{w s}} \sum_{\psi=0}^{n} \sum_{\theta=0}^{m} P_{C}(\psi, \theta, \varphi)
$$

where $n=2 \pi / \Delta \psi, m=\theta_{w s} / \Delta \theta$ and $P_{C}(\psi, \theta, \varphi)$ is defined by:

$$
P_{\mathrm{C}}(\psi, \theta, \varphi)\left\{\begin{array}{l}
0 \text { if } \lambda_{\mathrm{I}_{\mathrm{c}}}(\psi, \theta, \varphi)>0 \\
1 \text { if } \lambda_{\mathrm{I}_{\mathrm{c}}}(\psi, \theta, \varphi) \leqslant 0
\end{array}\right.
$$

The index, $\lambda_{\mathrm{I}_{\mathrm{c}}}=1-\left(\delta / \theta_{\max }\right)$, represents the ratio between the angle $\delta$ of the most inclined axis and the $\theta_{\max }$ maximum safety angle set to $75^{\circ}$.

The optimization problem was solved by genetic algorithm method under MATLAB software. The parameters used to run the GA are the following: a population with 50 individuals, a maximum of 100 generations with probabilities of crossover and of mutation respectively equal to $p_{c}=10 \%$ and $p_{m}=5 \%$. Using a computer with a CPU of $2 \mathrm{GHz}$ frequency, the algorithm is able to identify an optimal individual after 6 to $7 \mathrm{~min}$ of processing. This delay can be explained by the complexity of the inverse kinematic model [11] which needs to be resolved for each sampled point of the workspace.

\section{Results}

A Pareto front of only four solutions maximizing the objective function respecting tele-echography constraints is obtained. The solution retained, which is a compromise between the two opposed criteria of global dexterity and compactness, is the PROSIT 1 robot shown in Figure 4a and defined by the optimal design vector in Table 2 . This solution is the most compact with no singularity in the workspace centre. The prototype has been built and teleechography visual servoing experiments have been carried out. 
Table 2. The optimal design variables.

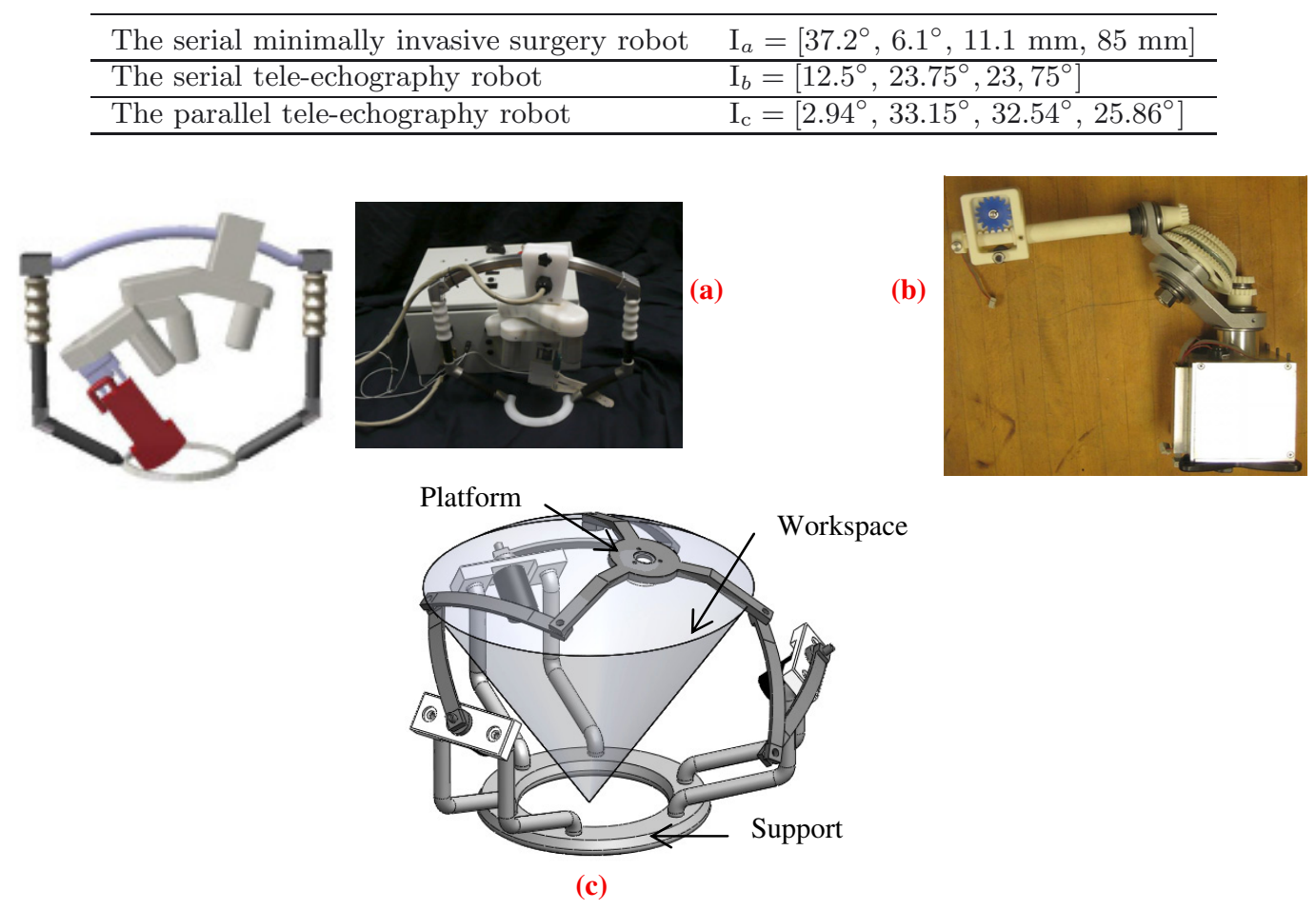

Fig. 4. (a) PROSIT-1 tele-echography robot - (b) Prototype MIS robot - (c) CAD illustration of the parallel spherical wrist.

The CAD illustration of the parallel spherical wrist, including its workspace, is shown in Figure 4c. It consists of a cone of $35^{\circ}$ apex angle.

For the case of the MIS robot, the optimization led to a family of solutions which satisfied the objectives and constraints. Using a set of weighting parameters which gave similar emphasis to each of the three criteria, the solution shown in Figure $4 \mathrm{~b}$ was obtained. The corresponding optimal design variables are shown in Table 2 . The prototype has undergone bench top and clinical experiments to validate its intended functionality.

\section{Conclusions}

This paper seeks to describe a dimensional synthesis process of spherical wrists for tool-guidance medical robots. Serial and parallel architectures were presented. Medical needs for two different applications (echography and minimally invasive surgery) were enumerated. Despite the differences between these applications in terms of medical needs, the mechanical specifications for different robots (kinematics, workspace, bulkiness) were the same or very similar. Multiple optimization criteria are combined linearly, as it is presented for different examples, in the objective function to meet practical needs in realistic applications. It is know that the most difficult point is the design criteria choice. The difference between the methods used for each spherical wrist optimization rested on the choices of criteria and constraints. Even though these methodologies may not give unique solutions, additional constraints appropriate to other medical applications can be used, allowing extension of these methods to a variety of medical robot design applications.

Acknowledgements. This research has been supported by "PROSIT" ANR project and by the Poitou-Charentes region 2007-2013 (program project 10 Images and interactivities), in partnership with the European Union (FEDER/ERDF, European Regional Development Fund).

\section{References}

[1] M. Lum, J. Rosen, M. Sinanan, B. Hannaford, Kinematic Optimization of a Spherical Mechanism for a Minimally Invasive Surgical Robot, Proceedings of IEEE In. Conf. on Rob. and Automations, Seattle, 2004, Vol. 1, pp. 36433648

[2] M. Lum, J. Rosen, M. Sinanan, B. Hannaford, Optimization of a Spherical Mechanism for Minimally Invasive Surgical Robot: Theoretical and Experimental Approaches, Trans. Biomedical Eng. 53 (2006)

[3] C. Gosselin, J. Angeles, The Optimum Kinematic Design of a Spherical 3 dof parallel Manipulator Journal of Mechanisms, Trans. Automation in Design 111 (1989)

[4] G. Alici, B. Shirinzadeh, Topology optimisation and singularity analysis of a 3-SPS parallel manipulator with a passive constraining spherical joint, Mech. Mach. Th. 39 (2004) $215-235$ 
[5] B. Shaoping, Optimum design of spherical parallel manipulators for a precribed workspace, Mech. Mach. Th. 45 (2010) 200-211

[6] A. Chaker, A. Mlika, M.A. Laribi, L. Romdhane, S. Zeghoul, Synthesis of a Spherical Parallel Manipulator for a Dexterous Medical Task, Front. Mech. Eng. 7 (2012) 150-162.

[7] A. Chaker, M.A. Laribi, S. Zeghloul, L. Romdhane, Design and Optimization of Spherical Parallel Manipulator as a Haptic Medical Device, the 37th Conf. of the IEEE IES, focusing on Ind. and manufacturing theory and applications of Electronics, controls, communications, instrumentation and computational intelligence (IECON 2011), 7-10 November 2011, Melbourne, Australia
[8] X. Zhang, C.A. Nelson, Multiple-Criteria Kinematic Optimization for the Design of Spherical Serial Mechanism Using Genetic Algorithm, ASME In. Design Engineering Tech. Conf., Montreal, 2010

[9] L. Nouaille, P. Vieyres, G. Poisson, Process of optimization for a 4 dof tele-echography robot, Robotica 30 (2012) $1131-1135$

[10] T. Essomba, M.A. Laribi, J.P. Gazeau, G. Poisson, S. Zeghloul, Design and optimization of a master-slave system for tele-echography application, in The ASME 2012 11th Biennial Conf. On Engineering Sys. Design And Analysis (ESDA2012), Nantes, France, 2012

[11] T. Essomba, M.A. Laribi, J.P. Gazeau, S. Zeghloul, G. Poisson, Contribution to the Design of a Robotized TeleEchography System, Front. Mech. Eng. 7 (2012) 135-149 